\title{
Developer-Proof Prevention of SQL Injections
}

\author{
Judicaël Courant ${ }^{[0000-0002-9880-2401]}$ \\ Orange Cyberdefense \\ Judicael.Courant@orangecyberdefense.com
}

\begin{abstract}
SQL queries, when written by unskilled or hurried developers for web applications, are prone to SQL injections. We propose an alternative approach for querying SQL databases that does not suffer from this flaw. Our approach is based on abstract syntax trees, lets developers build dynamic queries easily, and is easier to set up than an ORM tool. We provide a proof-of-concept in Java, but our approach can be extended to other languages.
\end{abstract}

Keywords: SQL injection · SQLi prevention · Dynamic queries · Code generation · Abstract syntax trees $\cdot$ Language-based security $\cdot$ Secure Coding $\cdot$ Software Security

\section{Introduction}

Can we prevent developers from writing programs vulnerable to SQL injections? Can we help them even if they do not care about security? These questions are of practical significance: SQL injections have been plaguing web applications for more than 20 years [19], yet they still rank first in application security flaws [21].

Here is a very simple example of vulnerable Java code:

$\mathrm{q}=$ "SELECT $*$ FROM atable WHERE token $=$ ' " + tok + "'";

rs $=$ connection.createStatement () . executeQuery $(q)$;

Listing 1: Example of vulnerable Java code

The code executes the following query, where the underlined placeholder is filled by the value of tok:

SELECT * FROM atable WHERE token $=$,

The intent of the programmer is to retrieve all rows whose column token is equal to the value of tok. Unfortunately, if an attacker gave " $x$ ' OR 1=1 -- " as a value for tok, the following query would be executed: 
SELECT * FROM atable WHERE token $=$ ' $\underline{x}$ ' OR $1=1--$ '

The semantics of the query significantly differs from the intent of the programmer: the WHERE clause is now the disjunction of two Boolean expressions, the second one, 1 = 1 , being a tautology.

Therefore, execution of the query now returns the whole table atable instead of some selected rows: if access to rows were supposedly restricted to users knowing the values of the token column, it is a severe vulnerability.

That is the essence of $S Q L$ injections: by supplying carefully crafted malicious data, the attacker could alter the SQL code generated by the programmer.

How should programmers write their code in order to ensure that it is immune to injections? Solutions exist: validating inputs, escaping harmful characters or using prepared statements [18].

Unfortunately, none of them alone covers the full spectrum of SQL injections and each of them is insecure if improperly used. Therefore, developers have to resort to a blend of these techniques. In order to make the right choice and write secure code, they have to be properly trained, and to be given enough time. In practice, they are not.

As a side-effect, source code security audits are complicated by this state of affairs: auditors have to inspect each and every query in order to make sure that it is immune to injection, which often involves tracking down the origin of each input. Source code analysis tools might help, but things can get quite involved, especially for second-order injections [4].

This article proposes a way to build queries that do not suffer from these limitations. Our contributions are the following:

- We reconsider using strings for building queries and propose that trees be used instead in section 2. We show how the example of listing 1 could be rewritten using a library for representing SQL queries as trees.

- We show how trees can be translated to standard queries, using a blend of techniques that developers would actually use in section 3 .

- We review classical injection attacks in section 4 and show, through examples, how our library prevents most of them.

- We implemented these ideas as a small Java library, available under a free software license [3]. We present it in section 5. Our library not only lets developers write secure queries but also prevents them from writing vulnerable ones.

- Our approach fills a gap between detection method based on parse trees and more heavyweight DOM-based methods relying on static typing to provide more guarantees, which we discuss in 6 .

- In conclusion, we suggest some possible future works that could help closing security limitations of existing ORM in section 7 .

\section{From Strings to Trees}

An interesting point of view over SQL injections is that they alter the syntax tree of the query. Figure 1 displays the expected shape of the syntax tree of the 
query built by listing 1 side-by-side with the actual tree built with the attackersupplied value for tok. The WHERE clause of the actual tree is quite different from the expected shape: in the expected shape, the WHERE clause was an equality between two terms (a single atomic proposition), whereas in the actual tree, the WHERE clause is the logical disjunction of two propositions.

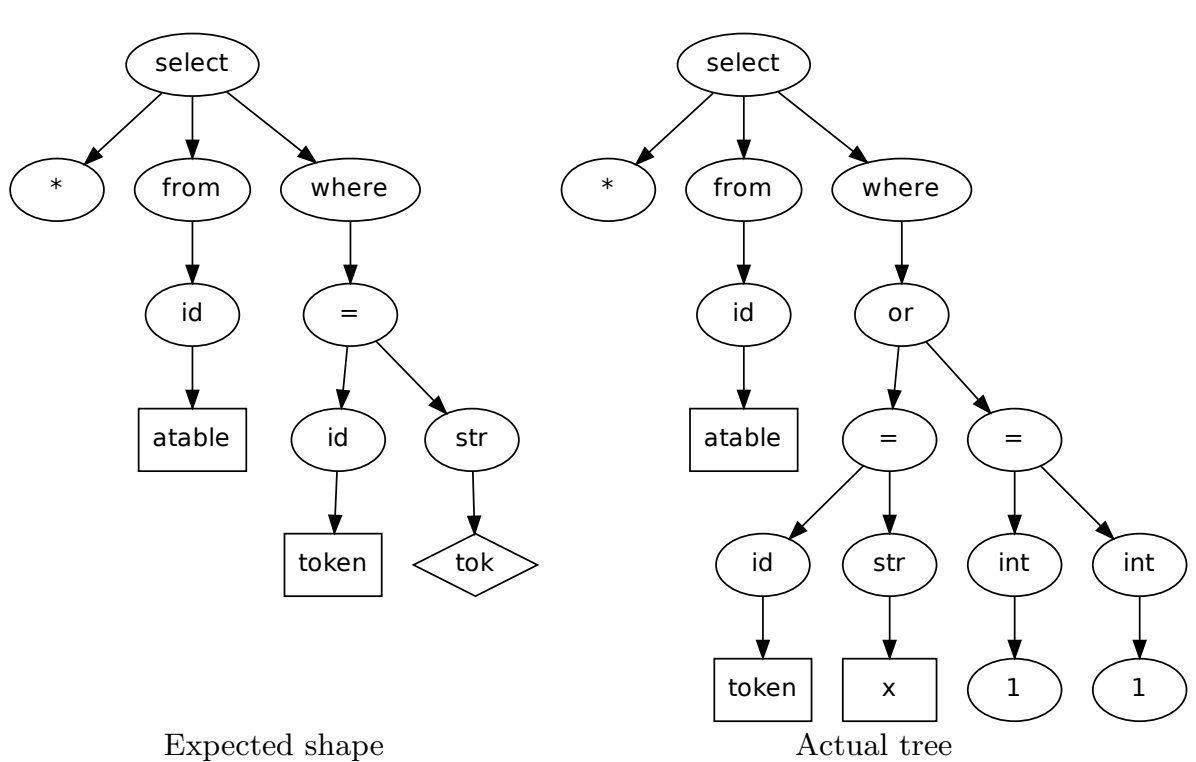

Fig. 1: Expected shape versus actual tree for listing 1

This situation is typical of a mismatch between what the programmer intended and what they actually programmed: the intent was to replace a leaf in a syntax tree, and string concatenation is the wrong way to do it.

Notice that generation of SQL queries is only a special case of code generation, which is a standard phase in compilers. Code generation is hardly ever done just by concatenating strings: most often, compilers use a large range of intermediate representations, mostly trees. Only at the end of the process is the last representation translated to strings. Therefore, we suggest to represent queries as trees.

We provide a small library for representing SQL syntax trees [3]. Actually, it provides an internal Domain Specific Language (DSL) that lets the user represent trees using a prefix notation (hence a somewhat Lispian taste).

Our initial example given in listing 1 can be rewritten with this internal DSL as shown in listing 2 . 


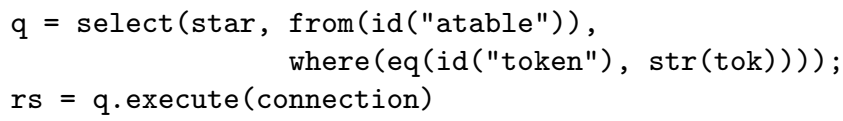

Listing 2: Initial example, rewritten using our library

In that code, select, from, id, where, eq, and str are static methods for building a tree. id and str build a tree respectively denoting an identifier and a string literal from a String, the other ones build a tree from one or more subtrees, and star is a constant leaf. As displayed in fig. 2, when the attacker supplies the same malicious value as previously, the shape of the tree is preserved.

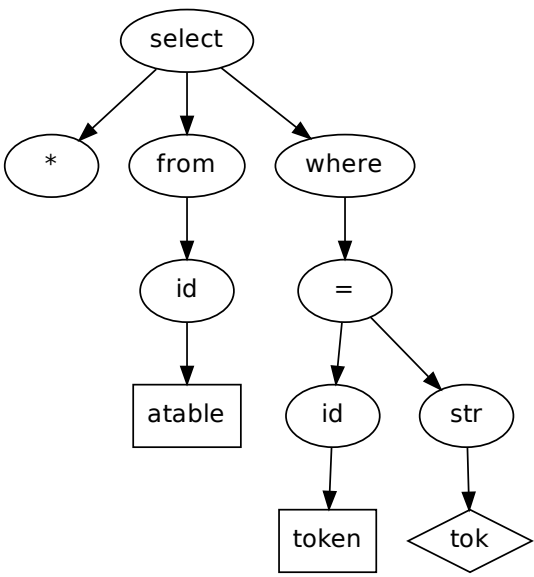

Expected shape

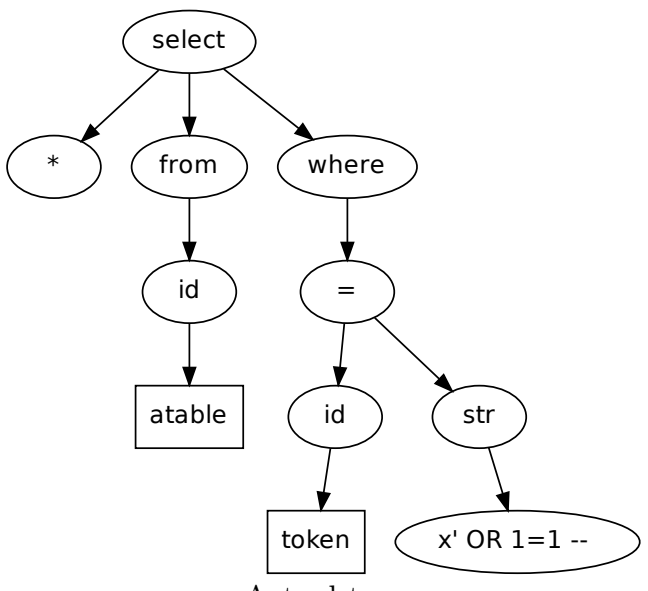

Actual tree

Fig. 2: Expected shape versus actual tree for listing 2

If there were some direct way to communicate the abstract syntax tree to the database we want to query, no injection could happen since, regardless of the value supplied for tok, the resulting tree $\mathrm{q}$ will have the intended shape, as displayed in fig. 2.

Unfortunately, as of 2020, the usual way to send a query to a database is to send a character string. Therefore, we have to compile the syntax tree to a string representing our query. 


\section{Compiling Trees to Strings}

In this section, we explain how we translate a syntax tree to a string that can be sent to an SQL database.

\subsection{Principle}

In order to make sure our code is immune to SQL injections, we have to make sure that the translation is faithful, in the following sense: if a tree $t$ is compiled into a string $c(t) s$ and that this string is again parsed into a tree $p(c(t))$, then $p(c(t))=t$.

The main difficulty here lies in the translation of the leafs of the trees, more precisely of identifiers and string literals.

As for string literals, we consider three options:

- Validating the value.

- Escaping dangerous characters.

- Using prepared statements

We reject the first option as it would be too dramatic a restriction. For instance, the string literal "L'île de Montréal" would be rejected since it contains the dangerous quote character. Yet it is a valid toponym in French. Likewise, the common Irish surname "O'Brian" would be rejected too.

Escaping dangerous characters is not an easy exercise as each database implementation escapes them in a slightly different way. We could use the library from the ESAPI project [24], but that would make our implementation depend on a non-standard library.

Fortunately, databases have a feature that provides a workaround for avoiding SQL injections at string literals: prepared statements. A prepared statement or parameterized query is a SQL query taking the form of a template in which some values are left unspecified: instead, they are replaced by placeholders, denoted by question marks in Java. The template and the actual values of the parameters are send separately to the database. The database driver encodes the parameters, so they cannot cause any injection.

That solution cannot apply when the input is supposed to be an identifier (a column name or a table name) though, since placeholders in prepared statements can only denote values, not identifiers. Therefore, another option must be considered. Restricting the set of accepted identifiers is much more reasonable than restricting the set of accepted literal strings. Thus, we ensure that the argument given to id is a valid identifier: we check that it is a non-empty sequence of alphanumeric characters starting with a letter and that it does not belong to the set of SQL keywords. We use an extensive list of 914 SQL keywords [7] in order to forbid not only keywords officially reserved in the SQL:2016 standard, but also some others reserved in common SQL databases. However, as for all blacklist approaches, it is not future-proof: if the SQL dialect understood by the database evolves, this list has to be updated. 


\section{$3.2 \quad$ Examples}

Let us first see how the translation from trees to strings prevents injection for listing 2. Suppose an attacker passes " $x$ ' OR 1=1 -- " as a value for tok. Then the query is the tree denoted by the following expression:

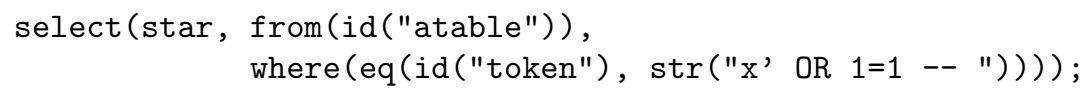

As explained in the previous section, the query is translated into a parameterized statement in which all literal strings (subtrees labeled by str) are turned into parameters:

SELECT $*$ FROM atable WHERE token $=$ ?

and the literal string " $\mathrm{x}$ ' OR 1=1 -- " is used as the value for the parameter. As explained in the previous section, no injection occurs: the database indeed looks for rows whose field token is equal to the literal string given by the attacker, whatever special characters it may contain.

As noted by [10, 13], prepared statements alone cannot prevent injections. For example, prepared statements cannot prevent injection at a column name or table name, since placeholder in prepared statements can only denote values, not identifiers. Consider for instance the following query:

SELECT pubcol

FROM WHERE timestamp > 1234

where the underlined part is supposed to be replaced by some user-supplied table name. The intent is that the user can read some public column of any table for which timestamp is large enough. An attacker could provide a malicious value in order to build the following query:

SELECT pubcol

FROM (SELECT seccol FROM atable) -- WHERE timestamp > 1234

Thus, bypassing the restriction over timestamp (as the WHERE clause has been turned into a comment) and over the columns.

Using our library, such a query would be coded as

select(id(pubcol), from(id(t)), where(gt(id("timestamp"), num(1234))))

Since the library validates that $t$ is a valid identifier, the attack would only raise an exception. Of course, that does not excuse developers from validating user input, unless all columns named pubcol in all tables are supposed to be readable by all users.

\section{SQL Injections Prevented by our Approach}

Classifications of SQL injection attacks can be found in [10, 23]. In [10], known injections attacks are presented and classified along two axis: mechanism and intent. In this section, we first discuss the mechanisms, then study how known attacks apply in our settings, and finally we discuss how much our setting restricts the goals an attacker could achieve. 


\subsection{Attack Mechanisms}

Mechanisms in [10], are the channels through which an attacker can inject malicious inputs. The main mechanisms are user inputs, cookies, server variables, and second-order injections (occurring when a payload is stored on the web server and used later on by the application to build a query). Assessing second-order injections is particularly challenging for static analysis approaches such as taint analysis [22]. However, in our approach, the mechanism axis is quite irrelevant since we do not distinguish between trusted and untrusted inputs: all inputs are untrusted.

\subsection{Known Attacks}

Known attacks, as listed in [10, 23], have the following impact in our setting:

- Tautologies, Union Query, Piggy-Backed Queries, Alternate Encodings, End of Line Comment, and OPENROWSET Result Retrieval all require the attacker to change the shape of the syntax tree of the query. Inference attacks (blind injections and timing attacks) also seem to require such a change. Therefore, they are prevented.

- Illegal/Logically Incorrect Queries are used by an attacker to gain information about the type and structure of the database. In order to mitigate such a risk, our library limits the details an attacker could learn by returning only a generic error containing no information in case an exception is raised by the database driver. Moreover, the attack surface is much reduced for the attacker. For instance, the attack proposed in [10] attempts to discover table names, by injecting some value. The attack fails in our setting for two reasons: since injecting values cannot provoke such errors, injection should happen at an identifier rather than at a value; moreover, a non-trivial expression is injected, which changes the shape of the syntax tree of the query. In order to succeed, an attacker would have to turn to more elaborated attacks at least.

- Stored Procedure attacks are a whole class of distinct attacks. Whereas all other attacks consist in sending a malicious payload to the application in order to get it to construct an unintended SQL query, attacks on stored procedures consist in having the application pass a malicious payload to the stored procedure, in order to get the stored procedure to construct an unintended SQL query.

As for stored procedure, listing 3 displays the authentication procedure proposed in [10]: A SQL query is dynamically constructed by concatenating strings, including the arguments Qusername and @password, which are supplied by the user of the stored procedure. Authentication would be bypassed by passing a value such as "admin' --" (if an admin account exists), and a database shutdown would be piggy-backed by passing the value " '; SHUTDOWN ; --", as suggested by the authors. Actually, the full spectrum of SQL injection attacks can potentially happen in the context of stored procedure. 


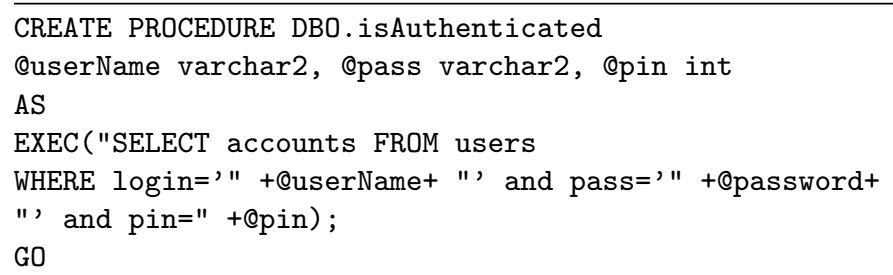

Listing 3: Vulnerable stored procedure

Our library does not prevent an attack against such a procedure, since it has no way to distinguish benign values from malicious ones by itself. Of course, a programmer using our library could validate user-supplied arguments before sending them to this procedure.

Arguably, counter-measures should be put in place in the database itself. Therefore, we consider that these stored procedure attacks are out of the scope of our approach. Possible counter-measures are:

- The designers of stored procedures should clearly specify which inputs are valid inputs and which are invalid.

- Developers of stored procedures should not trust inputs coming from the application: inputs should be validated in the procedure itself. As an additional benefit, that would prevent at once all vulnerable usages of corrected procedures, from all database users or clients.

- As much as possible, developers of stored procedures should refrain from building dynamic queries. The code above had no real reason to build a dynamic query. The static version, as displayed listing 4 , is immune to injection, simpler, shorter, and more readable.

- And of course, if the SQL dialect or the language used to define stored procedure is expressive enough to manipulate trees, our approach could be ported to that language. For instance, PostgreSQL allows stored procedures to be written in the Python language, and trees can easily be manipulated in Python.

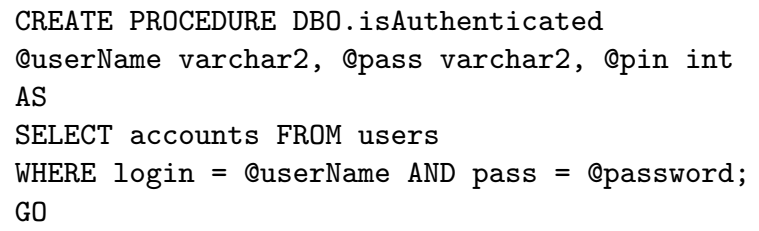


We note that [14] mentions an additional kind of injection regarding LIKE queries. Consider a query such as

SELECT * FROM messages WHERE subject LIKE $=$ ' $\%$

where the parameter is supposed to be filled by the concatenation of a usersupplied string $\mathrm{s}$ and a percent sign: the intent is to find all messages whose subject starts with s. Using our library, that would be coded as follows:

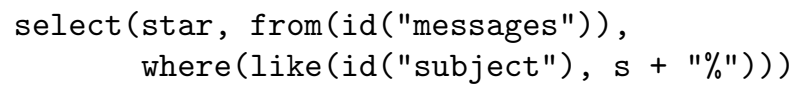

Unfortunately, the database engine might behave quite differently depending on the contents of $\mathbf{s}$. For instance, if $\mathrm{s}$ is the string "foo" and an index exists on column subject, then lookup takes time $O(\log n)$, where $n$ is the number of rows of the table. On the other hand, if an attacker supplies the string "\%foo", then the database can no longer take advantage of the index and lookup takes time $O(n)$. Such an injection is in fact an algorithmic complexity attack that could provoke a denial of service.

Interestingly, this attack does not change the abstract syntax tree of the query. Therefore, our library cannot prevent such an attack. The best countermeasure here seems to validate the value of $\mathbf{s}$.

\subsection{Attack Intents}

Possible malicious intents for an attacker are listed in [10]. We discuss here how effective our approach is against these intents (excluding stored procedure attacks for the reasons previously given):

- Identifying injectable parameters, performing database finger-printing, determining database schema are not completely prevented by our approach in principle since illegal/logically incorrect queries attacks are still possible. They are severely restricted however: all the actual attacks presented in [10] would fail in our setting since they all inject at a place where a value is expected and they all inject expressions that change the shape of the syntax tree.

- Performing denial of service might still be possible, but seems limited to LIKE queries. For instance, tables cannot be dropped.

- Extracting data, adding or modifying data, Bypassing authentication, executing remote commands, and performing privilege escalation do not seem possible in our setting since they all require to change the shape of the abstract syntax tree.

- As for evading detection, our approach does not consider that aspect.

Thus our approach seems to severely restrict the goals an attacker can achieve. 


\section{Implementation}

\section{$5.1 \quad$ Features}

Our approach is implemented in a small Java library [3]. The main features of our library are the following:

- It provides a small internal DSL for representing syntax trees.

- It provides a method to execute a query, represented as a tree, given a database connection.

- It is minimalistic: the shorter a code, the more likely its bugs and vulnerabilities can be corrected. Our library implements only a very small subset of SQL but it is so small (around 200 non-empty lines, not counting the list of the 900 reserved SQL keywords) that it can easily be adapted to other contexts.

- Database connection is achieved through the standard Connection class from the java.sql package. Thus issues regarding connections, drivers, and transactions do not have to be handled by our library.

- It is publicly available under a free software license [3].

\subsection{Organization}

Our implementation contains only seven classes. One is a custom exception class, and the six others are depicted in fig. 3. The class AST is the central class of our implementation. We use it in order to represent the abstract syntax tree of a query and to execute it (using the only public method execute).

Our implementation can be considered as an instance of the Interpreter design pattern, as proposed in [9]. Indeed, the authors notice that the pattern can also be used for implementing a compiler. In our implementation, the class CompiledStatement plays the role of the context and the method writeTo plays the role of the interpretation method.

A CompiledStatement object is used to build parameterized queries. The most relevant attributes and methods of the CompiledStatement class are given in listing 5. Calls to addToken gradually accumulate lexical tokens (identifier, keywords and numeric tokens) into the text of the parameterized query, implemented as the buffer tpl. Calls to addStringParam accumulate string literals by adding question marks in the template and corresponding string values into the list of parameters args.

Listing 6 displays the implementation of the execute method, from class AST, which you call in order to execute the query represented by the tree. The method writeTo, called by compile, is in charge of calling the methods addToken and/or addStringParam of $\mathrm{b}$ in order to build the actual Compiledstatement.

\subsection{Auditability}

During a source code audit, in order to ensure that a code that uses the standard Java classes java.sql. Statement is not vulnerable to SQL injections, you 


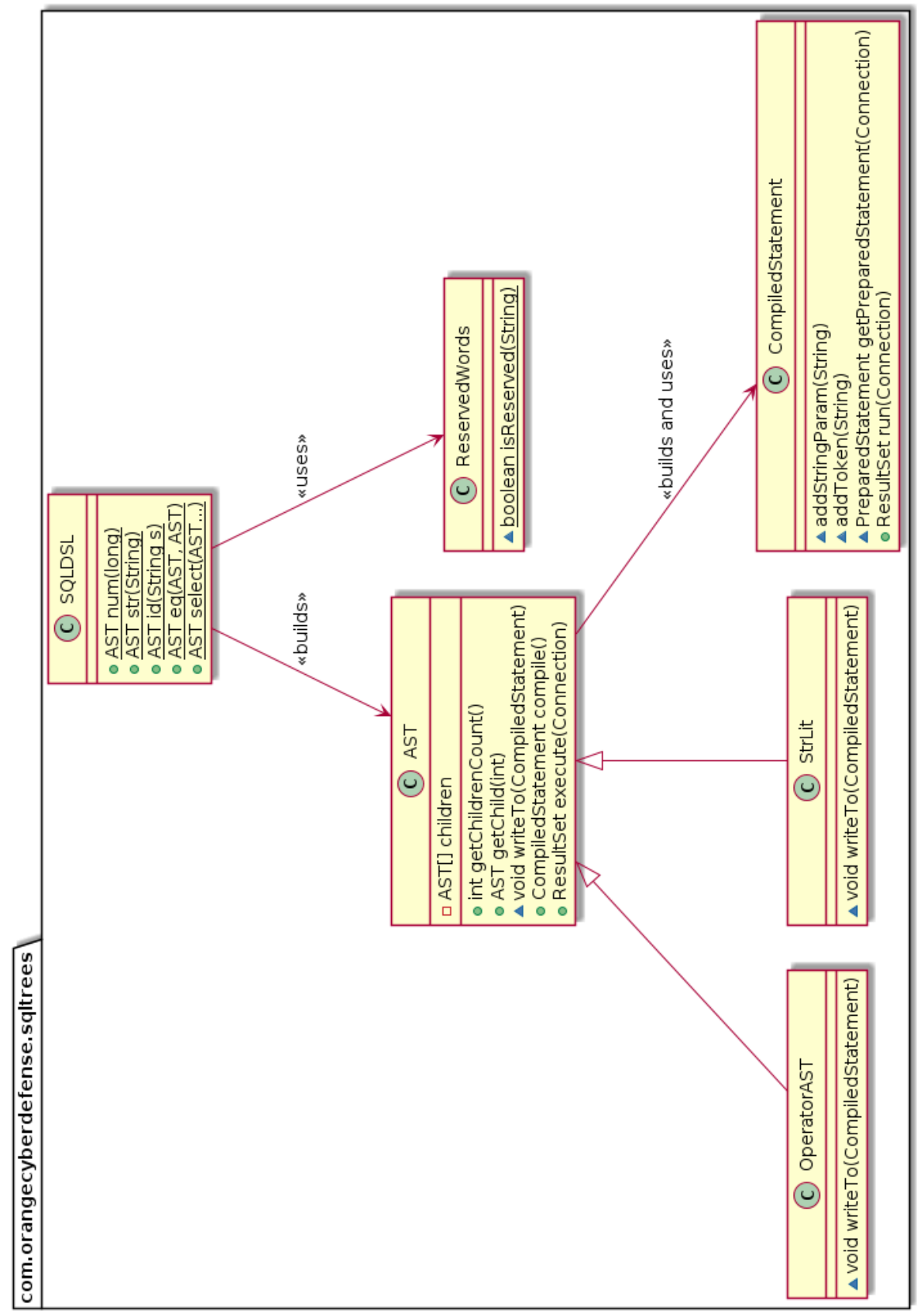

Fig. 3: Class Diagram 


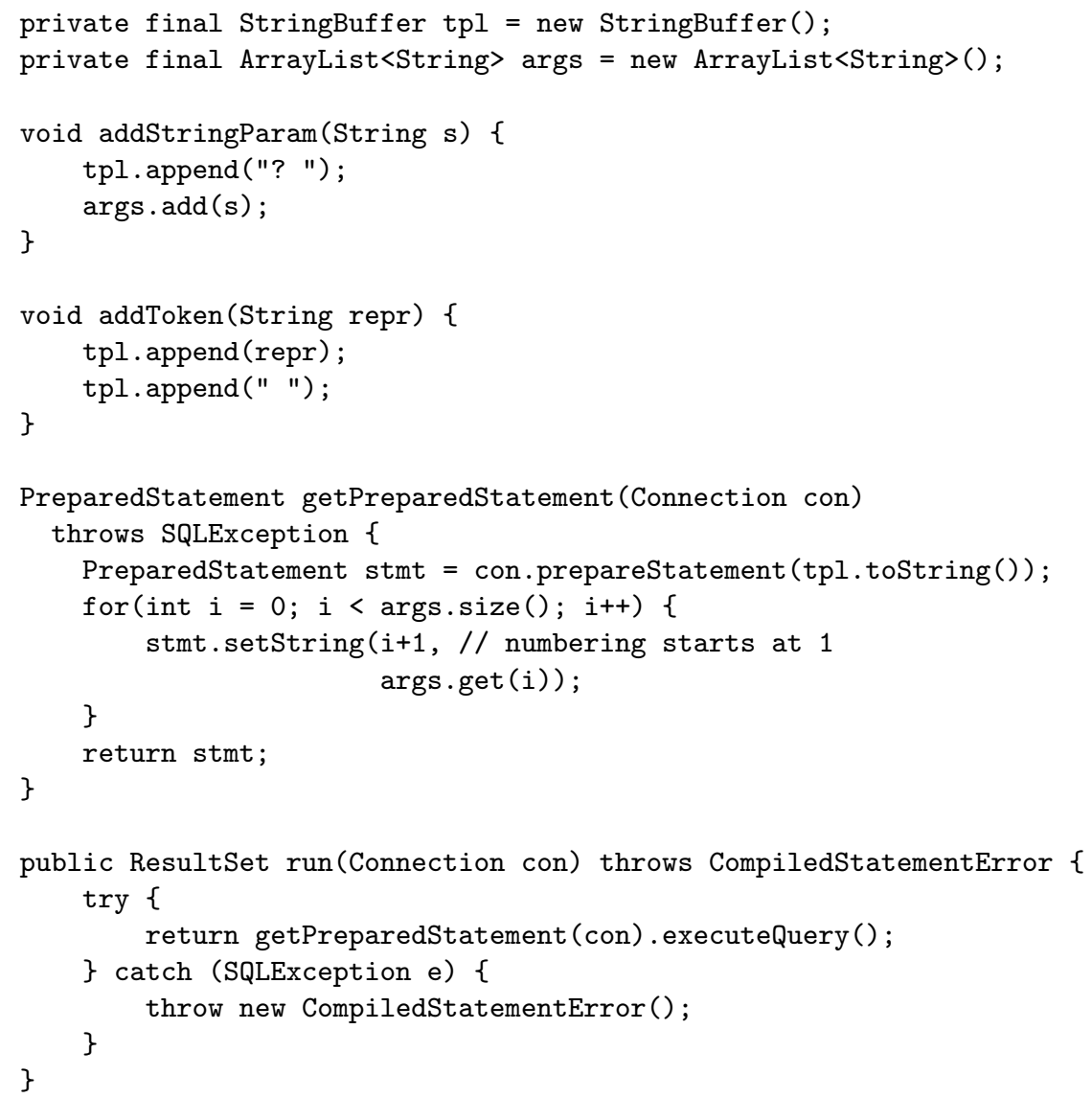

Listing 5: Main attributes and methods of CompiledStatement.java

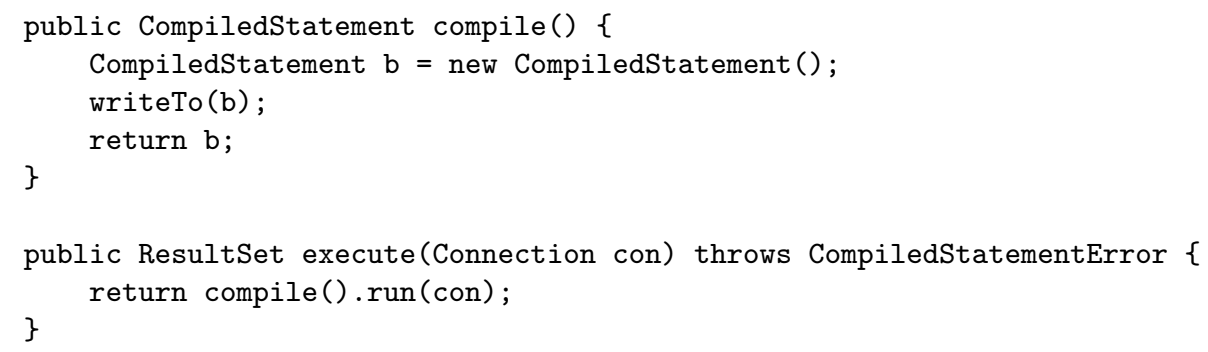

Listing 6: Methods from class AST for executing a syntax tree 
have to make sure that all untrusted inputs are validated, escaped, or passed as parameters. The issue even arises in practice when developers use prepared statements (see discussion about prepared statements in section 6.1). Such an analysis can be quite involved and can be time-consuming (even when using evolved static analysis tools).

We would like to replace these analysis by a much simpler criterion: if the code does neither use java.sql.Statement nor java.sql.PreparedStatement, but only our library, it is immune to most SQL injections.

Therefore, we must not only ensure that a code using our library as intended is secure. We must also guarantee that our library cannot be used in some insecure, unexpected ways. We identify the following cases as being problematic:

- Unexpected ways to create instances of the classes we provide.

- Modification of objects after creation time.

- Creation of new classes inheriting from a class from our library.

In order to prevent such issues, inheritance is restricted as much as possible (by declaring classes final), all classes but CompiledStatement construct immutable objects, and the visibility of classes, attributes, and methods are restricted as much as possible.

For instance, in order to let developers debug their queries, we make the class CompiledStatement public and provide public methods that reveal the contents of objects of that class. However, we ensure that these methods cannot alter the contents. In contrast, the methods addStringParam and addToken, which mutate the content of a CompiledStatement have their visibility restricted to the package.

We must note however a limitation of encapsulation in Java: an application can access non-public fields or methods via reflection, if you run it without any security manager or if you grant the appropriate permission to the security manager (namely ReflectPermission("suppressAccessChecks")).

\section{Related Works}

Injection-related vulnerabilities have been largely studied. Related works are numerous, but mostly fall in one of the following two categories:

Prevention works trying to reduce the gap between the source programming language and the language used for queries in order to make it easier to write queries.

Detection works trying to detect (and possibly stop) actual exploitation of the vulnerabilities.

\subsection{Prevention-Related Works}

Validating User Inputs Validating user-supplied inputs is generally recognized as a good idea. For instance, when a string of alphanumeric character is 
expected, a value containing a quote character can be rejected immediately. Unfortunately, although input validation is an inevitable part of secure coding, it is not enough to prevent injection. For instance, when a family name is expected, quote characters could not be rejected, without forbidding Irish surnames such as O'Brian.

Harmful Character Escaping Features for sanitizing inputs, like those provided by OWASP's Enterprise Security API [24] work. However, programmers have to explicitly use them on each user input. If they fail to do so, that will not be detected, neither at compile time nor at run time. And one omission is enough to make an application vulnerable. Therefore, using input sanitization as the only method for preventing SQL injections is risky. Moreover, in case of a source code security audit, each query must be reviewed.

Therefore, the PHP community used a mechanism that would systematically escape user inputs: magic quotes. Unfortunately, magic quotes apply indiscriminately on all user inputs, irrespective of the database. Moreover, developers depend on the sysadmins in charge of the server as they are the only persons who can enable it. Finally, magic quotes raise more issues than they solve. Accordingly, they were eventually removed from PHP [1].

Prepared Statements Prepared statements are the first defense option recommended by the OWASP project [18]. Unfortunately, they are only half a solution $[10,13]$ as they cannot protect against column or table injections.

Worse still, when improperly used, prepared statements are vulnerable to SQL injection attacks: since the template query is an ordinary string, nothing prevents developers from inserting unvalidated user inputs into the template part of the query.

Unfortunately, that happens in practice. Admittedly, when developers use prepared statements to search a database table on statically known criteria, their code is generally secure. But quite often, criteria are known only at execution time. Consider for example a product search for an online shop. In addition to the name of the product you are looking for, you may specify additional criteria (a maximum or minimum price, a category, etc.). Of course, the application must only consider criteria for which you gave a non empty value. Thus a SQL query has to be generated dynamically. Although it would be possible in theory to validate inputs, or to escape them, or to generate the right template together with the right list of parameters, it seems that, in practice, developers are much less rigorous. Criteria whose number and type are known at compile time are correctly passed as parameters, but the other ones tend to be appended to the template part. As a result, parameters known at compile-time cannot be injected, but the other ones are vulnerable if their contents have neither been validated nor escaped. We observed such a recurring anti-pattern in source code security audits. 
Programming Language Extensions SQLJ [8] and Embedded SQL [6] let you embed SQL queries in another programming language, such as Java, COBOL, Fortran, $\mathrm{C}$ or $\mathrm{C}++$. A preprocessor then checks your queries and translate them into your programming language. Such a way to write queries is quite natural for developers. However, although you can pass arguments at run-time, the structure of your queries has to be known at compile-time. This approach is thus restricted to static queries.

Moreover, on the practical side, that approach raises integration problems regarding existing tools: most IDE do not handle code extended with SQL constructs (syntax highlighting, contextual help, error location, etc.) and you have to twist the build tools in order to make them preprocess code before compilation.

Object-Relational Mapping Object-relational mapping can generate and execute SQL queries automatically. Tools like SQL DOM [16], Querydsl [20], and jOOQ [12] offer strong guarantees regarding execution a la "well-typed programs cannot go wrong" [17]: if the Java code compiles, then generated queries will be correct both regarding syntax and typing (no syntax error, no typing error). By contrast, our library does only guarantees that, if the abstract syntax tree is correct, then the generated query will be a syntactically correct representation of the tree.

ORM tools let you write more dynamic queries than embedded-SQL tools. Yet, these typed queries cannot provide access to all the SQL syntax. The situation is similar to the statically typed vs. untyped (or dynamically typed) dichotomy for programming languages: the statically typed approach is safer but less flexible than the untyped one. In order to compensate for this lack of flexibility, some ORM $[11,12]$ let developers write SQL queries as strings, which is a security risk if developers are not careful enough [5].

As a rule of thumb, adopting an ORM is a good decision at the very start of a project. Adopting it in on a legacy project is much more difficult from a software engineering point of view: ORM tools generally generate a data class for each table of the database. Developers then have to adapt their code to replace their homemade classes by the generated ones. In a project with tens or hundreds of tables and a large code base, this require good factoring skills and can be time-consuming. Therefore, for legacy projects in maintenance mode, switching to an ORM is often not an option. On the contrary, our approach requires little investment (you just have to understand how SQL syntax and our trees are related) and can be adopted gradually: you can replace each construction of Statement or PreparedStatement by a tree, one at a time.

\subsection{Detection-Related Works}

Static analysis techniques (such as taint analysis) can automatically analyze source code and detect some potential injection vulnerabilities at compile-time. 
However, leveraging such static analysis tools on legacy projects still looks difficult, because of the number of false positive that are initially raised and of skills needed to understand and correctly handle these alerts.

Several works $[15,2,22]$ suggest to detect injection attempts dynamically, using methods that go beyond usual Web Application Firewalls. Like us, they regard trees as a relevant representation for queries.

For instance, [2] and [22] suggest that developers would insert marks in their queries in order to delimit user-input data. Such marks let the authors build two abstract syntax trees: on the one hand, a model of the query the developers expected, and the other hand, a model of the query they actually built. The two trees are compared: a mismatch is the sign of injection attempt.

Using that method, developers can generate arbitrarily complex and dynamic queries. However, the security of the method relies on the goodwill and care of developers, as for the input sanitization method, mentioned earlier.

The method proposed by [15] for avoiding XSS when generating HTML code does not suffer from this limitation, since the source language they consider (Hop, a Lisp-like programming language) lets you represent abstract syntax trees easily: you just have to write the abstract syntax of the HTML fragment you want to build as a tree. Therefore, you do not need to put any mark in order to extract a model: the tree you wrote is the model. Moreover the authors prove that their method is correct from a semantic point of view. Although the authors are concerned with HTML code generation whereas we are concerned with SQL code generation, our work is close to theirs. However, instead of generating code that is faithful to the tree, like we did (which means that dangerous characters have to be watched for), they chose to generate the HTML code without handling dangerous characters, and to parse the resulting text in order to detect a potential injection attempt.

\section{Conclusion}

Manipulating trees in Java is quite easy and is a simple, effective way to build dynamic queries that are immune to most injections. Tree structures are easily implemented as immutable structures. In functional programming, it is common knowledge that you can easily guarantee properties on such structures, once they are correctly encapsulated. More specifically, in our case, they help to ensure that developers will not build vulnerable queries.

Finally, we would like to extend this work in the following directions:

- From a theoretical point of view, we would like to compare our approach to [22]. Can we adapt the formal definition of injections given in that article and mathematically prove that our approach is secure?

- We could also provide libraries for other languages than Java. However, in languages with looser encapsulation facilities, it might be more difficult to ensure that such a library is developer-proof. We would like to study how language features help (or hinder) the development of such a library. We conjecture already that a strong static type system helps. 
- We would like to extend our approach to other similar injections problems: XSS, LDAP injections, shell command injections, etc.

- Our library is only a proof of concept as it handles only a tiny subset of SQL at the moment. We would like to grow our implementation in order to make it usable in real projects. All contributions are of course very welcome!

- Our work has the potential to access all SQL syntax, which is more difficult for ORM tools. We would like to see whether direct accesses to SQL queries as strings provided by ORM could be replaced by accesses to our abstract syntax trees.

\section{Acknowledgments}

Claire Vacherot and the anonymous referees provided valuable feedback that helped improve the article.

\section{References}

[1] Sebastian Bergmann, Arne Blankerts, and Stefan Priebsch. Why Magic Quotes are gone in PHP 7. Aug. 2017. URL: https://thephp.cc/news/ 2017/08/why-magic-quotes-are-gone-in-php7.

[2] Gregory T. Buehrer, Bruce W. Weide, and Paolo A. G. Sivilotti. "Using parse tree validation to prevent SQL injection attacks". In: Proceedings of the International Workshop on Software Engineering and Middleware (SEM) at Joint FSE and ESEC. 2005, pp. 106-113.

[3] Judicaël Courant. Sqltrees: a Secure, Developper-Proof, Java Library for Querying SQL Databases. 2020. URL: https : //github . com/OrangeCyberdefense/sqltrees.

[4] Johannes Dahse and Thorsten Holz. "Static Detection of Second-Order Vulnerabilities in Web Applications". In: Proceedings of the 23rd USENIX Security Symposium, San Diego, CA, USA, August 20-22, 2014. Ed. by Kevin Fu and Jaeyeon Jung. URL: https://www . usenix.org/conference/ usenixsecurity14/technical-sessions/presentation/dahse. USENIX Association, 2014, pp. 989-1003.

[5] Lukas Eder. Never Concatenate Strings With jOOQ. jOOQ blog. URL: https: //blog. jooq.org/2020/03/04/never-concatenate-stringswith-jooq/. Mar. 2020.

[6] Wikipedia, The Free Encyclopedia. Embedded SQL. Mar. 2020.

[7] Wikipedia, The Free Encyclopedia. SQL Reserved Words. Mar. 2020.

[8] Wikipedia, The Free Encyclopedia. SQLJ. Mar. 2020.

[9] E. Gamma et al. Design Patterns: Elements of Reusable Object-Oriented Software. Addison-Wesley Professional Computing Series. Pearson Education, 1994. ISBN: 978-0-201-63361-0.

[10] William G.J. Halfond, Jeremy Viegas, and Alessandro Orso. "A Classification of SQL-Injection Attacks and Countermeasures". In: Proceedings of the International Symposium on Secure Software Engineering. Washington D.C., USA, Mar. 2006. 
[11] Hibernate project. URL: http://hibernate.org/.

[12] jOOQ website. URL: https://www.jooq.org/.

[13] Bill Karwin. SQL Injection Myths and Fallacies. URL: https : / / www . percona.com/sites/default/files/WEBINAR-SQL-Injection-Myths . pdf. 2012.

[14] Jayant Shekhar Khaleel Ahmad and K.P. Yadav. "Classification of SQL Injection Attacks". In: VSRD Technical \& Non-Technical Journal I (4) (May 2010), pp. 235-242.

[15] Zhengqin Luo, Tamara Rezk, and Manuel Serrano. "Automated Code Injection Prevention for Web Applications". In: Theory of Security and Applications - Joint Workshop, TOSCA 2011, Saarbrücken, Germany, March 31 - April 1, 2011, Revised Selected Papers. Ed. by Sebastian Mödersheim and Catuscia Palamidessi. Vol. 6993. Lecture Notes in Computer Science. Springer, 2011, pp. 186-204. DOI: 10.1007/978-3-642-27375-9_11.

[16] R. A. McClure and I. H. Kruger. "SQL DOM: compile time checking of dynamic SQL statements". In: Proceedings. 27th International Conference on Software Engineering, 2005. ICSE 2005. 2005, pp. 88-96.

[17] Robin Milner. "A theory of type polymorphism in programming". In: Journal of Computer and System Sciences 17 (1978), pp. 348-375.

[18] OWASP project. SQL Injection Prevention Cheat Sheet. URL: https: / / cheatsheetseries.owasp.org/cheatsheets/SQL_Injection_Prevention_ Cheat_Sheet.html. 2020.

[19] Rain Forest Puppy. "NT Web Technology Vulnerabilities". In: Phrack Magazine 8.54 (Dec. 1998). URL: http://phrack.org/issues/54/8. html.

[20] Querydsl website. URL: http://www.querydsl.com/.

[21] Andrew van der Stock et al. OWASP Top 10 - 2017. 2017. URL: https : //web . archive .org/web/20200406122129/https : //owasp .org/wwwpdf-archive/OWASP_Top_10-2017_(en) .pdf .pdf.

[22] Zhendong Su and Gary Wassermann. "The essence of command injection attacks in web applications". In: Proceedings of the 33rd ACM SIGPLANSIGACT Symposium on Principles of Programming Languages, POPL 2006. Ed. by J. Gregory Morrisett and Simon L. Peyton Jones. Charleston, South Carolina, USA: ACM, 2006, pp. 372-382. DOI: 10.1145/1111037. 1111070.

[23] San-Tsai Sun et al. "Classification of SQL Injection Attacks". University of Columbia, Term Project. URL: https : / / courses . ece.ubc.ca/ cpen442/term_project/reports/2007-fall/Classification_of_SQL_ Injection_Attacks.pdf. 2007.

[24] Kevin Wall and Matt Seil. The OWASP Enterprise Security API. URL: https://web.archive.org/web/20200331100823/https://owasp.org/ www-project-enterprise-security-api/. 